Article

\title{
On a Certain Generalized Functional Equation for Set-Valued Functions
}

\author{
Yaroslav Bazaykin, Dušan Bednařík*iD, Veronika Borůvková and Tomáš Zuščák \\ Department of Mathematics, Faculty of Science, University of Hradec Králové, \\ 50003 Hradec Králové, Czech Republic; yaroslav.bazaykin@uhk.cz (Y.B.); veronika.boruvkova@uhk.cz (V.B.); \\ tomas.zuscak@uhk.cz (T.Z.) \\ * Correspondence: dusan.bednarik@uhk.cz; Tel.: +420-49-333-2840
}

Received: 15 October 2020; Accepted: 17 December 2020; Published: 19 December 2020

Abstract: The aim of the paper is to generalize results by Sikorska on some functional equations for set-valued functions. In the paper, a tool is described for solving a generalized type of an integral-functional equation for a set-valued function $F: X \rightarrow c c(Y)$, where $X$ is a real vector space and $Y$ is a locally convex real linear metric space with an invariant metric. Most general results are described in the case of a compact topological group $G$ equipped with the right-invariant Haar measure acting on $X$. Further results are found if the group $G$ is finite or $Y$ is Asplund space. The main results are applied to an example where $X=\mathbb{R}^{2}$ and $Y=\mathbb{R}^{n}, n \in \mathbb{N}$, and $G$ is the unitary group $U(1)$.

Keywords: integral-functional equation; set-valued function; topological vector space; compact topological group

MSC: 54C60; 39B52; 39B55

\section{Introduction}

Jordan and von Neumann in 1935 [1] gave characterizations of inner product spaces among normed vector spaces $(X,\|\cdot\|)$ which lead to the functional equation in the form

$$
f(x+y)+f(x-y)=2 f(x)+2 f(y), x, y \in X .
$$

Fréchet in 1935 [2] obtained a characterization of normed spaces with inner product in connection with the following functional equation:

$$
f(x+y+z)+f(x)+f(y)+f(z)=f(x+y)+f(x+z)+f(y+z), x, y, z \in X .
$$

Later, Drygas in 1987 [3] studied the following functional equation now called the Drygas equation in order to characterize quasi-inner product spaces:

$$
f(x+y)+f(x-y)=2 f(x)+f(y)+f(-y), x, y \in X .
$$

Interested readers can find useful information about such kinds of equations in the book by Kannappan [4] or by Aczél and Dhombres [5]. Sikorska in [6] considers analogies of these equations for unknown set-valued functions. It has been shown in [6] that these equations can be transformed into the form which can be viewed as special cases of the equation

$$
F(x)+\frac{\beta-1}{2 \beta^{2}} F(-\beta x)=\frac{\beta+1}{2 \beta^{2}} F(\beta x) .
$$


In addition, the Cauchy orthogonal equation for set-valued functions can be solved as a special form of the previous equation. These types of equations are used in physics, statistics, and mathematical programming. We attempted to further generalize the equation and hopefully cover more special cases of set-valued functional equations, which one can encounter, for instance, in mathematical economics.

Sikorska in her paper [6] introduced some methods for solving certain types of functional equations with the unknown being a set-valued mapping. She used properties of the Hausdorff metric and the so-called Hukuhara difference. In her article, we can find the results for solving the following functional equation:

$$
F(x)+\frac{\beta-1}{2 \beta^{2}} F(-\beta x)=\frac{\beta+1}{2 \beta^{2}} F(\beta x),
$$

with $\beta$ natural number, $\beta \neq 1,(X,+)$ group uniquely divisible by $\beta, x \in X,(Y, d)$ a locally convex linear metric space with an invariant metric, and $F$ is a set-valued mapping from $X$ to the family of non-empty compact, convex subsets of $Y$.

We consider the following generalization of Equation (1). Take the $G$ finite group with identity element $e$. It is convenient for us to fix an enumeration of elements of $G$ starting from $e: G=\left\{\gamma_{0}=\right.$ $\left.e, \gamma_{1}, \ldots, \gamma_{n}\right\}$, where $|G|=n+1$. Let $G$ act on $X$ by linear transformations. Consider the following functional equation for set-valued functions:

$$
F(x)+\frac{\beta-1}{(n+1) \beta^{2}} \sum_{\gamma \in G, \gamma \neq e} F(\beta \gamma x)=\frac{1+n \beta}{(n+1) \beta^{2}} F(\beta x) .
$$

The case $G=\mathbb{Z}_{2}$ yields Equation (1). Equation (2) can be written in the form:

$$
F(x)+\frac{\beta-1}{(n+1) \beta^{2}} \sum_{\gamma \in G} F(\beta \gamma x)=\frac{1}{\beta} F(\beta x) .
$$

Finally, consider one more generalization of Equation (3). Let $G$ be a compact topological group equipped with the right-invariant Haar measure $\mu$. Let $|G|=\int_{G} d \mu(\sigma)$; G acts on $X$ by linear transformations. Consider the following integral-functional equation for a real number $D>1$ :

$$
F(x)+\frac{D-1}{D^{2}} \frac{1}{|G|} \int_{G} F(D \sigma x) d \mu(\sigma)=\frac{1}{D} F(D x) .
$$

The article concentrates on solving Equation (4), using the Hukuhara difference similarly to Sikorska in [6].

\section{Preliminary Results}

We present some notions and results used in the article. All the results can be found in [6], and the first two originate in [7]. In the following, for a topological vector space $Y$, we denote by $c(Y), c c(Y)$, and $b c l(Y)$ the family of all non-empty compact, non-empty compact convex, and non-empty bounded closed subsets of $Y$, respectively.

Lemma 1. Let $A, B$, and $C$ be sets in a topological vector space. Supposing $B$ is closed and convex, $C$ is bounded, non-empty, and $A+C \subset B+C$. Then, $A \subset B$.

Lemma 2. If $A, B$ are closed and convex sets in a topological vector space and $C$ is bounded and non-empty, then $A+C=B+C$ implies $A=B$.

For $(Y, d)$ metric space, define the Hausdorff distance between $A, B \in b c l(Y)$ as in [6]. The space $b c l(Y)$ equipped with the Hausdorff distance is a metric space, and we call $d$ the Hausdorff metric. On this metric space, we consider a set convergence for a sequence of sets $\left(A_{n}\right)_{n \in \mathbb{N}} \subset b c l(Y)$. We define 
its limit $A, A_{n} \rightarrow A$, meaning that the sequence of sets $\left(A_{n}\right)_{n \in \mathbb{N}}$ converges to $A$ with respect to the Hausdorff metric.

Lemma 3. Let $(Y, d)$ be a metric linear space. Then,

1. for all $A_{n}, B_{n}, A, B \in \operatorname{bcl}(Y), n \in \mathbb{N}$, if $A_{n} \rightarrow A$ and $B_{n} \rightarrow B$; then, $A_{n}+B_{n} \rightarrow A+B$;

2. if $d$ is invariant, $A \in \operatorname{bcl}(Y)$, and $\left(\alpha_{n}\right)_{n \in \mathbb{N}}$ is a sequence of real numbers converging to $\alpha \in \mathbb{R}$, then $\alpha_{n} A \rightarrow \alpha A$.

Let $Y$ be a real topological vector space. If we have $A, B \in c c(Y)$, then, in general, we cannot find $X \in c c(Y)$ such that $B+X=A$. If such $X$ exists, then we denote it $A-B$ and call it the Hukuhara difference of $A$ and $B$. The following lemma will be used in the proof of existence of the Hukuhara difference.

Lemma 4 ([6]). Let $(Y, d)$ be a locally convex metric space with an invariant metric. Let $A, A_{n}, B, B_{n} \in c c(Y)$ such that $A_{n} \rightarrow A$ and $B_{n} \rightarrow B$. Let, moreover, for each $n \in \mathbb{N}$, a Hukuhara difference $A_{n}-B_{n}$ to exist. Then, there exists the Hukuhara difference $A-B$ and $A_{n}-B_{n} \rightarrow A-B$.

In [6], Sikorska states:

Theorem 1. Let $\beta$ be a positive integer different from 1 , let $(X,+)$ be a group uniquely divisible by $\beta$, and let $(Y, d)$ be a locally convex linear metric space with an invariant metric. Assume that $F: X \rightarrow c c(Y)$ satisfies

$$
F(x)+\frac{\beta-1}{2 \beta^{2}} F(-\beta x)=\frac{\beta+1}{2 \beta^{2}} F(\beta x), x \in X .
$$

Then, $F$ is of the form $F=a+G$, where $a: X \rightarrow Y$ is an odd function satisfying $a(\beta x)=\beta a(x)$ for all $x \in X$, and $G: X \rightarrow c c(Y)$ is an even set-valued function with $G(\beta x)=\beta^{2} G(x)$ for all $x \in X$. Moreover, such $a$ representation is unique.

We can generalize the theorem for an integral-functional Equation (4).

\section{Compact Topological Group Case for Set-Valued Functions}

Let $G$ be a compact topological group equipped with the right-invariant Haar measure $\mu$. Letting $|G|=\int_{G} d \mu(\sigma), G$ acts on a real vector space $X$ by linear transformations. Consider the following integral-functional equation for a set-valued function $F: X \rightarrow c c(Y)$, where $Y$ is a locally convex real linear metric space with an invariant metric (we assume that $D$ is a real number and $D>1$ ):

$$
F(x)+\frac{D-1}{D^{2}} \frac{1}{|G|} \int_{G} F(D \sigma x) d \mu(\sigma)=\frac{1}{D} F(D x) .
$$

We can find a particular type of solutions as follows. Let functions $A: X \rightarrow c c(Y)$ and $b: G \times X \rightarrow Y$ satisfy the following conditions:

$$
\begin{array}{r}
A(\gamma x)=A(x), \\
A(D x)=D^{2} A(x)
\end{array}
$$

and

$$
\begin{array}{r}
b(\gamma, \sigma x)=b(\gamma \sigma, x)-b(\sigma, x), \\
b(\gamma, D x)=D b(\gamma, x),
\end{array}
$$


for all $x \in X, \gamma, \sigma \in G$. Let

$$
h(x)=\frac{1}{|G|} \int_{G} b(\gamma, x) d \mu(\gamma)
$$

exist for all $x \in X$.

Lemma 5. Let $G$ be a compact topological group equipped with the right-invariant Haar measure $\mu$. Let $X$ be a real vector space. Let $Y$ be a locally convex real linear metric space with an invariant metric. Let $D$ be a real number and $D>1$. Let functions $A: X \rightarrow c c(Y)$ and $b: G \times X \rightarrow Y$ satisfy conditions (7) and (8). Then, $A(x)$ and $h(x)$ defined by (9) are solutions of Equation (6). Consequently, $F(x)=A(x)+h(x)$ is a solution of (6). In addition,

$$
\begin{gathered}
\int_{G} h(\sigma x) d \mu(\sigma)=0, \\
h(D x)=D h(x),
\end{gathered}
$$

for all $x \in X$.

Proof. First of all, we prove (10):

$$
\begin{array}{r}
\int_{G} h(\sigma x) d \mu(\sigma)=\frac{1}{|G|} \int_{G}\left(\int_{G} b(\gamma, \sigma x) d \mu(\gamma)\right) d \mu(\sigma)= \\
=\frac{1}{|G|} \int_{G}\left(\int_{G} b(\gamma \sigma, x) d \mu(\gamma)-\int_{G} b(\sigma, x) d \mu(\gamma)\right) d \mu(\sigma)= \\
=\frac{1}{|G|} \int_{G}\left(\int_{G} b(\gamma, x) d \mu(\gamma)-|G| b(\sigma, x)\right) d \mu(\sigma)= \\
=\frac{1}{|G|} \int_{G}\left(\int_{G} b(\gamma, x) d \mu(\gamma)\right) d \mu(\sigma)-\int_{G} b(\sigma, x) d \mu(\sigma)= \\
=\int_{G} b(\gamma, x) d \mu(\gamma)-\int_{G} b(\sigma, x) d \mu(\sigma)=0,
\end{array}
$$

and

$$
h(D x)=\frac{1}{|G|} \int_{G} b(\gamma, D x) d \mu(\gamma)=\frac{1}{|G|} \int_{G} D b(\gamma, x) d \mu(\gamma)=D h(x) .
$$

Then, direct substitution of $A$ in (6) gives

$$
\begin{array}{r}
A(x)+\frac{D-1}{D^{2}} \frac{1}{|G|} \int_{G} A(D \sigma x) d \mu(\sigma)= \\
=A(x)+\frac{D-1}{D^{2}} \frac{1}{|G|} \int_{G} A(D x) d \mu(\sigma)= \\
=A(x)+\frac{D-1}{D^{2}} A(D x)= \\
=A(x)+(D-1) A(x)=D A(x)=\frac{1}{D} A(D x) .
\end{array}
$$

Analogously,

$$
\begin{array}{r}
h(x)+\frac{D-1}{D^{2}} \frac{1}{|G|} \int_{G} h(D \sigma x) d \mu(\sigma)=h(x)+\frac{D-1}{D} \frac{1}{|G|} \int_{G} h(\sigma x) d \mu(\sigma)= \\
h(x)=\frac{1}{D} h(D x) .
\end{array}
$$

The next theorem shows that the inverse statement is also true with some limitations. 
Theorem 2. Let $G$ be a compact topological group equipped with the right-invariant Haar measure $\mu$. Let $X$ be a real vector space. Let $Y$ be a locally convex real linear metric space with an invariant metric. Let $D$ be a real number and $D>1$. Assume that a set-valued function $F: X \rightarrow c c(Y)$ satisfies Equation (6) for all $x \in X$. Then, there exists a set-valued function $\tilde{F}: X \rightarrow c c(Y), \tilde{F}(x) \subset F(x)$ for all $x \in X$, which also satisfies Equation (6) for all $x \in X$ such that there exist a set-valued function $A: X \rightarrow c c(Y)$ and a single-valued function $h: X \rightarrow Y$ and $\tilde{F}$ can be expressed in the following way:

$$
\tilde{F}(x)=A(x)+h(x), x \in X
$$

and functions $A$ and $h$ satisfy conditions (7) and (10). Furthermore, expression (11) is unique. The function $h$ has a unique form

$$
h(x)=\frac{1}{|G|} \int_{G} b(\gamma, x) d \mu(\gamma)
$$

where the function $b: G \times X \rightarrow Y$ satisfies the condition (8).

Proof. We state that, if $F(x)$ is a solution of (6), then

$$
F(x)+\frac{D^{n}-1}{D^{2 n}} \frac{1}{|G|} \int_{G} F\left(D^{n} \sigma x\right) d \mu(\sigma)=\frac{1}{D^{n}} F\left(D^{n} x\right), n \in \mathbb{N} .
$$

We will prove (13) by induction over $n$. For $n=1,(13)$ coincides with Equation (6). Let (13) hold for some $n \in \mathbb{N}$. Substituting $x:=D^{n} x$ in Equation (6), we obtain:

$$
F\left(D^{n} x\right)+\frac{D-1}{D^{2}} \frac{1}{|G|} \int_{G} F\left(D^{n+1} \sigma x\right) d \mu(\sigma)=\frac{1}{D} F\left(D^{n+1} x\right) \text {. }
$$

Then,

$$
\begin{array}{r}
F(x)+\frac{D^{n}-1}{D^{2 n}} \frac{1}{|G|} \int_{G} F\left(D^{n} \sigma x\right) d \mu(\sigma)+\frac{D-1}{D^{n+2}} \frac{1}{|G|} \int_{G} F\left(D^{n+1} \sigma x\right) d \mu(\sigma)= \\
\frac{1}{D^{n}} F\left(D^{n} x\right)+\frac{D-1}{D^{n+2}} \frac{1}{|G|} \int_{G} F\left(D^{n+1} \sigma x\right) d \mu(\sigma)=\frac{1}{D^{n+1}} F\left(D^{n+1} x\right) .
\end{array}
$$

Now, substitute $x:=\gamma x$ in (14), integrate over all $\gamma \in G$, and multiply by $\left(D^{n}-1\right) /\left(D^{2 n}|G|\right)$. We obtain

$$
\begin{array}{r}
\frac{D^{n}-1}{D^{2 n}} \frac{1}{|G|} \int_{G} F\left(D^{n} \gamma x\right) d \mu(\gamma)+ \\
+\frac{(D-1)\left(D^{n}-1\right)}{D^{2(n+1)} \frac{1}{|G|^{2}} \int_{G}(}\left(\int_{G} F\left(D^{n+1} \sigma \gamma x\right) d \mu(\sigma)\right) d \mu(\gamma)= \\
\frac{D^{n}-1}{D^{2 n+1}} \frac{1}{|G|} \int_{G} F\left(D^{n+1} \gamma x\right) d \mu(\gamma) .
\end{array}
$$

For certain $\gamma \in G$, consider the map $g_{\gamma}: G \rightarrow G, g_{\gamma}(x)=x \gamma$ for every $x \in G$. Clearly, $G-\gamma$ is a bijection for every $\gamma \in G$. We can write:

$$
\int_{G} F(\sigma \gamma x) d \mu(\sigma)=\int_{G} F(\sigma x) d \mu(\sigma),
$$

on condition of the existence of one of the integrals for given $x \in X$. Similarly,

$$
\begin{array}{r}
\int_{G}\left(\int_{G} F\left(D^{n+1} \sigma \gamma x\right) d \mu(\sigma)\right) d \mu(\gamma)=\int_{G}\left(\int_{G} F\left(D^{n+1} \sigma x\right) d \mu(\sigma)\right) d \mu(\gamma)= \\
|G| \int_{G} F\left(D^{n+1} \sigma x\right) d \mu(\sigma)=|G| \int_{G} F\left(D^{n+1} \gamma x\right) d \mu(\gamma) .
\end{array}
$$


Hence, we can rewrite (16)

$$
\begin{array}{r}
\frac{D^{n}-1}{D^{2 n}} \frac{1}{|G|} \int_{G} F\left(D^{n} \gamma x\right) d \mu(\gamma)+\frac{(D-1)\left(D^{n}-1\right)}{D^{2(n+1)}} \frac{1}{|G|} \int_{G} F\left(D^{n+1} \gamma x\right) d \mu(\gamma)= \\
\frac{D^{n}-1}{D^{2 n+1}} \frac{1}{|G|} \int_{G} F\left(D^{n+1} \gamma x\right) d \mu(\gamma) .
\end{array}
$$

Using (19) and trivial decomposition

$$
\frac{D-1}{D^{n+2}}=\frac{D-1}{D^{2(n+1)}}+\frac{(D-1)\left(D^{n}-1\right)}{D^{2(n+1)}}
$$

we can rewrite (15) as

$$
\begin{aligned}
& \frac{1}{D^{n+1}} F\left(D^{n+1} x\right)=F(x)+\frac{D^{n}-1}{D^{2 n}} \frac{1}{|G|} \int_{G} F\left(D^{n} \sigma x\right) d \mu(\sigma)+ \\
& \frac{(D-1)\left(D^{n}-1\right)}{D^{2(n+1)}} \frac{1}{|G|} \int_{G} F\left(D^{n+1} \sigma x\right) d \mu(\sigma)+\frac{(D-1)}{D^{2(n+1)}} \frac{1}{|G|} \int_{G} F\left(D^{n+1} \sigma x\right) d \mu(\sigma)= \\
& F(x)+\frac{D^{n}-1}{D^{2 n+1}} \frac{1}{|G|} \int_{G} F\left(D^{n+1} \sigma x\right) d \mu(\sigma)+\frac{(D-1)}{D^{2(n+1)}} \frac{1}{|G|} \int_{G} F\left(D^{n+1} \sigma x\right) d \mu(\sigma)= \\
& F(x)+\frac{D^{n+1}-1}{D^{2(n+1)}} \frac{1}{|G|} \int_{G} F\left(D^{n+1} \sigma x\right) d \mu(\sigma),
\end{aligned}
$$

which completes the proof of Equation (13) by induction.

Now, substitution $x:=x / D^{n}$ in (13) and multiplication by $D^{n}$ gives

$$
D^{n} F\left(\frac{x}{D^{n}}\right)+\frac{D^{n}-1}{D^{n}} \frac{1}{|G|} \int_{G} F(\sigma x) d \mu(\sigma)=F(x) .
$$

Equation (20) implies an existence of the Hukuhara difference

$$
F(x)-\frac{D^{n}-1}{D^{n}} \frac{1}{|G|} \int_{G} F(\sigma x) d \mu(\sigma)=D^{n} F\left(\frac{x}{D^{n}}\right) .
$$

Then, using Lemmas 3 and 4, we obtain that the following Hukuhara difference exists:

$$
F(x)-\frac{1}{|G|} \int_{G} F(\sigma x) d \mu(\sigma) .
$$

Now, define functions $A, H: X \rightarrow c c(Y)$ as follows:

$$
\begin{array}{r}
A(x)=\frac{1}{|G|} \int_{G} F(\sigma x) d \mu(\sigma), \\
H(x)=F(x)-\frac{1}{|G|} \int_{G} F(\sigma x) d \mu(\sigma) .
\end{array}
$$

By the definition of the Hukuhara difference, we have $F(x)=A(x)+H(x)$. Then,

$$
A(\gamma x)=\frac{1}{|G|} \int_{G} F(\sigma \gamma x) d \mu(\sigma)=\frac{1}{|G|} \int_{G} F(\sigma x) d \mu(\sigma)=A(x),
$$

for all $x \in X, \gamma \in G$. 


$$
\begin{aligned}
D^{2} F(x)+(D-1) A(D x) & =D^{2}\left(F(x)+\frac{D-1}{D^{2}} \frac{1}{|G|} \int_{G} F(D \sigma x) d \mu(\sigma)\right) \\
& =D F(D x) .
\end{aligned}
$$

Substituting $x:=\gamma x$ in (22) and averaging over $\gamma \in G$, we obtain

$$
D^{2} A(x)+(D-1) A(D x)=D A(D x)=A(D x)+(D-1) A(D x) .
$$

Using Lemma 2 Equation (23) implies

$$
D^{2} A(x)=A(D x)
$$

Going further, we have

$$
F(\gamma x)=H(\gamma x)+\frac{1}{|G|} \int_{G} F(\sigma \gamma x) d \mu(\sigma)=H(\gamma x)+\frac{1}{|G|} \int_{G} F(\sigma x) d \mu(\sigma) .
$$

Hence,

$$
F(\gamma x)=H(\gamma x)+A(x)
$$

Averaging (25) over $\gamma \in G$, we obtain

$$
A(x)=\frac{1}{|G|} \int_{G} H(\gamma x) d \mu(\gamma)+A(x),
$$

which implies

$$
\frac{1}{|G|} \int_{G} H(\gamma x) d \mu(\gamma)=\{0\}
$$

Going further, we have

$$
\begin{array}{r}
H(D x)+D^{2} A(x)=H(D x)+A(D x)=F(D x)=D F(x)+ \\
\frac{D-1}{D} \frac{1}{|G|} \int_{G} F(D \sigma x) d \mu(\sigma)=D H(x)+D A(x)+\frac{D-1}{D} A(D x)= \\
=D H(x)+D A(x)+\left(D^{2}-D\right) A(x)=D H(x)+D^{2} A(x),
\end{array}
$$

which implies

$$
H(D x)=D H(x)
$$

for all $x \in X$.

Using induction, we can easily show that the following equality is also true

$$
H\left(D^{m} x\right)=D^{m} H(x),
$$

for all $x \in X, m \in \mathbb{Z}$.

Now, we construct a single-valued function $h: X \rightarrow Y$ such that $h(x) \in H(x)$ for all $x \in X$ and moreover $h(D x)=D h(x)$. We define a relation $\sim$ on the set $X$ as follows: Let $x_{1}, x_{2} \in X$. Then, $x_{1} \sim x_{2}$ iff there exists $k \in \mathbb{Z}$ such that $x_{1}=D^{k} x_{2}$. This relation is obviously an equivalence. Furthermore, we consider the decomposition of the set $X$ into classes by the relation $\sim$. Let us consider a particular class of decomposition by the relation $\sim$; we denote it $S$. Let us select a particular element $r \in S$ and choose a value $h(r)$ so that $h(r) \in H(r)$. Obviously, such a selection does not necessarily have to be unambiguous. Furthermore, for any $x \in S$, there exists $m \in \mathbb{Z}$ such that $x=D^{m} r$. For these $x, m$, 
we put $h(x)=D^{m} h(r)$. Using (29), it follows that $H(x)=H\left(D^{m} r\right)=D^{m} H(r)$. Hence, $h(x) \in H(x)$. Thus, we can determine $h(x)$ for any $x \in X$ by this way.

Therefore, we can further consider a single-valued function $h: X \rightarrow Y$ with these properties:

$$
\begin{gathered}
h(x) \in H(x), \\
h(D x)=D h(x),
\end{gathered}
$$

for all $x \in X$.

Moreover, (26) implies immediately

$$
\int_{G} h(\gamma x) d \mu(\gamma)=0
$$

For every $\gamma \in G$ and every $x \in X$, we put $b(\gamma, x)=h(x)-h(\gamma x)$. Then, we easily infer

$$
\begin{array}{r}
\frac{1}{|G|} \int_{G} b(\gamma, x) d \mu(\gamma)= \\
\frac{1}{|G|} \int_{G} h(x) d \mu(\gamma)-\frac{1}{|G|} h(\gamma x) d \mu(\gamma)=h(x), \\
b(\gamma \sigma, x)-b(\sigma x)= \\
h(x)-h(\gamma \sigma x)-h(x)+h(\sigma x)=b(\gamma, \sigma x) \\
b(\gamma, D x)= \\
h(D x)-h(\gamma D x)=D h(x)-D h(\gamma x)=D b(\gamma, x)
\end{array}
$$

Equations (21) and (24) prove (7) for function $A$. Equations (34) and (35) prove (8) for function $b$. Equation (33) proves (9) for function $h$. By Lemma 5, function $\tilde{F}(x)=A(x)+h(x)$ solves the Equation (6). Now, we have for every $x \in X$ that $\tilde{F}(x) \subset F(x)$. Lemma 5 or Equations (31) and (32) prove (10) for function $h$.

Moreover, we can find that

$$
\begin{aligned}
& \frac{1}{|G|} \int_{G} \tilde{F}(\sigma x) d \mu(\sigma)=\frac{1}{|G|} \int_{G}(A(\sigma x)+h(\sigma x)) d \mu(\sigma)= \\
& \quad=\frac{1}{|G|} \int_{G} A(x) d \mu(\sigma)+\frac{1}{|G|} \int_{G} h(\sigma x) d \mu(\sigma)=A(x)
\end{aligned}
$$

therefore,

$$
A(x)=\frac{1}{|G|} \int_{G} F(\sigma x) d \mu(\sigma)=\frac{1}{|G|} \int_{G} \tilde{F}(\sigma x) d \mu(\sigma) .
$$

To prove uniqueness, suppose $\tilde{F}(x)=\tilde{A}(x)+\tilde{h}(x)$ for some functions $\tilde{A}: X \rightarrow c c(Y)$ and $\tilde{h}: X \rightarrow Y$ satisfying (7) and (10). Then,

$$
\begin{array}{r}
A(x)=\frac{1}{|G|} \int_{G} \tilde{F}(\sigma x) d \mu(\sigma)=\frac{1}{|G|} \int_{G}(\tilde{A}(\sigma x)+\tilde{h}(\sigma x)) d \mu(\sigma)= \\
=\frac{1}{|G|} \int_{G} \tilde{A}(x) d \mu(\sigma)+\frac{1}{|G|} \int_{G} \tilde{h}(\sigma x) d \mu(\sigma)=\tilde{A}(x) .
\end{array}
$$

Then,

$$
A(x)+\tilde{h}(x)=\tilde{A}(x)+\tilde{h}(x)=\tilde{F}(x)=A(x)+h(x),
$$


which implies

$$
\tilde{h}(x)=h(x)
$$

It remains for us to prove the uniqueness of representation (12). To do this, assume

$$
h(x)=\frac{1}{|G|} \int_{G} \tilde{b}(\sigma, x) d \mu(\sigma)
$$

where $\tilde{b}$ satisfies (8). Then,

$$
\begin{array}{r}
b(\gamma, x)=h(x)-h(\gamma x)=\frac{1}{|G|} \int_{G}(\tilde{b}(\sigma, x)-\tilde{b}(\sigma, \gamma x)) d \mu(\sigma)= \\
=\frac{1}{|G|} \int_{G}(\tilde{b}(\sigma, x)-\tilde{b}(\sigma \gamma, x)+\tilde{b}(\gamma, x)) d \mu(\sigma)=\frac{1}{|G|} \int_{G} \tilde{b}(\sigma, x) d \mu(\sigma)- \\
\frac{1}{|G|} \int_{G} \tilde{b}(\sigma \gamma, x) d \mu(\sigma)+\tilde{b}(\gamma, x)=\tilde{b}(\gamma, x),
\end{array}
$$

for all $x \in X, \gamma \in G$.

\section{Further Results}

Note that, in Theorem 1, Sikorska proved that every function $F$, solution of (5), can be uniquely expressed as the sum of a set-valued function $G$ and a single-valued function $a$. We proved in Theorem 2 that every function $F$, solution of (6) contains a function $\tilde{F}$, and also a solution of (6), which can be expressed as the sum of a set-valued function $A$ and a single-valued function $h$. Furthermore, for a certain solution $f$, there can exist more such functions $\tilde{F}$. In the following section, we concentrate on some of the cases where $F=\tilde{F}$ holds.

First, let us consider the case of finite group $G$ and the measure $\mu$ of an arbitrary subset $\tilde{G}$ of a set $G$. The measure $\mu$ gives the number of the members of the set. Then, for the set-valued function $K: G \rightarrow \mathcal{P}(Y)$, we can set

$$
\int_{G} K(\gamma) d \mu(\gamma)=: \sum_{\gamma \in G} K(\gamma)
$$

Lemma 6. Let the set-valued function $F: X \rightarrow c c(Y)$ be a solution of Equation (6) for each $x \in X$, where $G$ is finite. Let $\tilde{F}: X \rightarrow c c(Y)$ be a solution of (6) constructed according to Theorem 2. Then, $F(x)=\tilde{F}(x)$ for each $x \in X$.

Proof. In accordance with the proof of Theorem 2, we obtain

$$
A(x)=\frac{1}{|G|} \sum_{\sigma \in G} F(\sigma x),
$$

for every $x \in X$. The Formula (26) gives us

$$
\frac{1}{|G|} \sum_{\gamma \in G} H(\gamma x)=\{0\}
$$

for each $x \in X$. Since $G$ is finite, (39) immediately gives that the set $H(x)$ is a singleton for every $x \in X$. Considering, as in Theorem 2, $\tilde{F}(x)=A(x)+h(x)$, with $h(x) \in H(x)$, the function $h$ is unique and $F(x)=\tilde{F}(x)$ for each $x \in X$. 
Theorem 3. Let $G$ be a finite compact topological group, where $|G|=n+1$. Let $X$ be a real vector space. Let $Y$ be a locally convex real linear metric space with an invariant metric. Let $D$ be a real number and $D>1$. Assume that a set-valued function $F: X \rightarrow c c(Y)$ satisfies the equation

$$
F(x)+\frac{D-1}{(n+1) D^{2}} \sum_{\gamma \in G} F(D \gamma x)=\frac{1}{D} F(D x) \text {. }
$$

for all $x \in X$. Then, there exists a set-valued function $A: X \rightarrow \operatorname{cc}(Y)$ satisfying

$$
A(D x)=D^{2} A(x), A(\gamma x)=A(x)
$$

for all $x \in X, \gamma \in G$ and a single-valued function $h: X \rightarrow Y$ satisfying

$$
h(D x)=D h(x), \sum_{\sigma \in G} h(\sigma x)=0
$$

for all $x \in X, \gamma \in G$ such that $F$ can be expressed in the following way:

$$
F(x)=A(x)+h(x), x \in X,
$$

Furthermore, this expression is unique. The function $h$ has a unique form

$$
h(x)=\frac{1}{n+1} \sum_{\gamma \in G} b(\gamma, x)
$$

where the function $b: G \times X \rightarrow Y$ satisfies the condition

$$
b(\gamma, \sigma x)=b(\gamma \sigma, x)-b(\sigma, x), b(\gamma, D x)=D b(\gamma, x)
$$

for all $x \in X, \gamma, \sigma \in G$

Proof. The theorem follows immediately from Theorem 2 and Lemma 6.

Let us now consider a more general case. Suppose $Y$ is an Asplund space, i.e., Banach space, whose topological dual space $Y^{*}$ is separable. In this case, we will show that, for any $x \in X$, it holds that $F(\gamma x)=\tilde{F}(\gamma x)$ for $\mu$-almost every $\gamma \in G$.

Lemma 7. Let $\Gamma$ be a measurable correspondence, i.e., set-valued mapping of some measurable space $(G, \mathcal{A})$ into $\operatorname{cc}(Y)$, then the functions

$$
\begin{aligned}
& G \ni \gamma \rightarrow \sup _{y \in \Gamma(\gamma)}\left\{y^{*}(y)\right\} \\
& G \ni \gamma \rightarrow \inf _{y \in \Gamma(\gamma)}\left\{y^{*}(y)\right\}
\end{aligned}
$$

are measurable for each $y^{*} \in Y^{*}$.

Proof. We repeat the proof of Lemma 1 of [8]. Fix $y^{*} \in Y^{*}$ and $t \in \mathbb{R}$. If

$$
A_{t}=\left\{\gamma \in G: \sup _{y \in \Gamma(\gamma)}\left\{y^{*}(y)\right\}>t\right\}
$$

it follows that the the graph $\operatorname{Gr}(\Gamma)$ of the correspondence $\Gamma$ belongs to the product $\sigma$-algebra $\mathcal{A} \otimes \mathcal{B}(Y)$, where $\mathcal{B}(Y)$ denotes the Borel $\sigma$-algebra in $Y$. Then, also

$$
\left\{(\gamma, y) \in \operatorname{Gr}(\Gamma): y^{*}(y)>t\right\}
$$


belongs to $\sigma$-algebra $\mathcal{A} \otimes \mathcal{B}(Y)$. Therefore, the set $A_{t}$ must belong to the $\sigma$-algebra $\mathcal{A}$ being a projection of the previous set on the set $G$.

The proof of the following lemma can be found in [8].

Lemma 8. Let $\Gamma$ be a measurable correspondence of a measurable space $G$ into $c c(Y)$ and

$$
\int_{G} \Gamma(\gamma) d \mu(\gamma) \neq \varnothing
$$

Then, the following equalities hold for each $y^{*} \in Y^{*}$ :

$$
\begin{aligned}
\sup _{y \in \int_{G} \Gamma(\gamma) d \mu(\gamma)}\left\{y^{*}(y)\right\} & =\int_{G} \sup _{y \in \Gamma(\gamma)}\left\{y^{*}(y)\right\} d \mu(\gamma), \\
\inf _{y \in \int_{G} \Gamma(\gamma) d \mu(\gamma)}\left\{y^{*}(y)\right\} & =\int_{G} \inf _{y \in \Gamma(\gamma)}\left\{y^{*}(y)\right\} d \mu(\gamma) .
\end{aligned}
$$

The following Lemma is an easy consequence of the definition of the integral. Hence, the proof is omitted.

Lemma 9. Let $G$ be a measurable space and $f: G \rightarrow \mathbb{R}$ be a nonnegative function. Let $t>0$ be real and $A_{t}=\{\gamma \in G: f(\gamma) \geq t\}$. Then, the following holds:

$$
\mu\left(A_{t}\right) \leq \frac{1}{t} \int_{A_{t}} f(\gamma) d \mu(\gamma) \leq \frac{1}{t} \int_{G} f(\gamma) d \mu(\gamma) .
$$

Theorem 4. Suppose that the set-valued function $F: X \rightarrow c c(Y)$ solves Equation (6) for each $x \in X$, where $Y$ is Asplund space (which means that the dual space $Y^{*}$ is assumed separable.) Let $\tilde{F}: X \rightarrow c c(Y)$ be the solution of (6) constructed according to Theorem 2 . Then, for every $x \in X$, it holds that

$$
F(\gamma x)=\tilde{F}(\gamma x)
$$

for $\mu$-almost every $\gamma \in G$.

Proof. Similarly to the Theorem 2,

$$
\begin{array}{r}
F(x)=A(x)+H(x), \\
\tilde{F}(x)=A(x)+h(x),
\end{array}
$$

where

$$
\begin{array}{r}
A(x)=\frac{1}{|G|} \int_{G} F(\sigma x) d \mu(\sigma), \\
h(x) \in H(x)
\end{array}
$$

for each $x \in X$. It suffices to show that, for each $x \in X$, there exists a set $\mathcal{N} \subset G$ such that $\mu(\mathcal{N})=0$ and $H(\gamma x)$ is a singleton for every $\gamma \in G \backslash \mathcal{N}$.

From (26), we obtain

$$
\int_{G} H(\gamma x) d \mu(\gamma)=\{0\}
$$


for each $x \in X$. Now, for $y^{*} \in Y^{*}, x \in X$ denote

$$
\begin{aligned}
& s_{y^{*}, x}(\gamma)=\inf _{y \in H(\gamma x)}\left\{y^{*}(y)\right\}, \\
& S_{y^{*}, x}(\gamma)=\sup _{y \in H(\gamma x)}\left\{y^{*}(y)\right\} .
\end{aligned}
$$

Since for every $\gamma \in G, x \in X$ the set $H(\gamma x)$ lies in $c c(Y), y^{*}(y)$ attains its minimum and maximum on the set $H(\gamma x)$ (so the functions can be defined). Thus, with the help from (41), the functions $s_{y^{*}, x}: G \rightarrow \mathbb{R}$ a $S_{y^{*}, x}: G \rightarrow \mathbb{R}$ are correctly defined.

Furthermore, as the function $G \ni \gamma \rightarrow H(\gamma x) \in c c(Y)$ is for each $x \in X$ supposed to be measurable, integrable, and set-valued, the functions $s_{y^{*}, x}$ and $S_{y^{*}, x}$ are, according to Lemma 7, measurable as well. For every $y^{*} \in Y^{*}$, Lemma 8 yields:

$$
\begin{aligned}
& 0=\sup _{y \in \int_{G} H(\gamma x) d \mu(\gamma)=\{0\}}\left\{y^{*}(y)\right\}=\int_{G} S_{y^{*}, x}(\gamma) d \mu(\gamma), \\
& 0=\inf _{y \in \int_{G} H(\gamma x) d \mu(\gamma)=\{0\}}\left\{y^{*}(y)\right\}=\int_{G} s_{y^{*}, x}(\gamma) d \mu(\gamma) .
\end{aligned}
$$

Moreover, consider for every $y^{*} \in Y^{*}, x \in X$ function $f_{y^{*}, x}: G \rightarrow \mathbb{R}$ defined:

$$
f_{y^{*}, x}(\gamma)=S_{y^{*}, x}(\gamma)-s_{y^{*}, x}(\gamma), \gamma \in G
$$

Obviously, for each $\gamma \in G, f_{y^{*}, x}(\gamma) \geq 0$ holds. Clearly, the function $f_{y^{*}, x}$ is measurable for each $y^{*} \in Y^{*}$, $x \in X$; therefore, (42) for arbitrary fixed $y^{*} \in Y^{*}, x \in X$ :

$$
\begin{array}{r}
\int_{G} f_{y^{*}, x}(\gamma) d \mu(\gamma)= \\
=\int_{G} S_{y^{*}, x}(\gamma) d \mu(\gamma)-\int_{G} s_{y^{*}, x}(\gamma) d \mu(\gamma)=0 .
\end{array}
$$

As a result of Lemma 9, $f_{y^{*}, x} \equiv 0$ holds $\mu$-almost everywhere on $G$ for every $y^{*} \in Y^{*}, x \in X$. Moreover, because of the separability of dual $Y^{*}$, there exists a countable dense set $\left\{y_{i}^{*}\right\} \subset Y^{*}$. This gives, for every $i \in \mathbb{N}$ and $x \in X$, a set that exists $\mathcal{N}_{i} \subset G$ of zero measure, such that $f_{y_{i}^{*}, x} \equiv 0$ na $G \backslash \mathcal{N}_{i}$. Then, $\mathcal{N}=\bigcup_{i \in \mathbb{N}} \mathcal{N}_{i}$ holds the following: $f_{y_{i}^{*}, x} \equiv 0$ na $G \backslash \mathcal{N}$ for each $i \in \mathbb{N}$, and $x \in X$ and $\mathcal{N}$ is of zero measure.

We now prove that, for each $x \in X$ and $\gamma \in G \backslash \mathcal{N}$, the set $H(\gamma x)$ s a singleton. On the contrary, suppose there exist $x \in X$ and $\gamma \in G \backslash \mathcal{N}$, such that $H(\gamma x)$ contains two different elements $y_{1}, y_{2} \in Y$. Then, $y_{1}-y_{2} \neq 0$. Thus, $0<\left\|y_{1}-y_{2}\right\|$ and there exists $\hat{y}^{*} \in Y^{*}$, such that $\hat{y}^{*}\left(y_{1}-y_{2}\right)=1$. Now, let $0<\varepsilon<\frac{1}{\left\|y_{1}-y_{2}\right\|}$. Thus, there is $y_{i_{0}}^{*} \in\left\{y_{i}^{*}\right\}$ such that $\left\|\hat{y}^{*}-y_{i_{0}}^{*}\right\|_{*}<\varepsilon$. It gives us:

$$
\begin{array}{r}
\left|\hat{y}^{*}\left(y_{1}-y_{2}\right)-y_{i_{0}}^{*}\left(y_{1}-y_{2}\right)\right|=\left|\left(\hat{y}^{*}-y_{i_{0}}^{*}\right)\left(y_{1}-y_{2}\right)\right| \leq \\
\leq\left\|\hat{y}^{*}-y_{i_{0}}^{*}\right\|_{*}\left\|y_{1}-y_{2}\right\|<\varepsilon\left\|y_{1}-y_{2}\right\| .
\end{array}
$$

Then, we obtain

$$
1-\left|y_{i_{0}}^{*}\left(y_{1}-y_{2}\right)\right|<\varepsilon\left\|y_{1}-y_{2}\right\|<1
$$

i.e.,

$$
0<1-\varepsilon\left\|y_{1}-y_{2}\right\|<\left|y_{i_{0}}^{*}\left(y_{1}-y_{2}\right)\right| .
$$

Without loss of generality, we can suppose $y_{i_{0}}^{*}\left(y_{1}-y_{2}\right)>0$. This yields

$$
y_{i_{0}}^{*}\left(y_{2}\right)<y_{i_{0}}^{*}\left(y_{2}\right)+\left(1-\varepsilon\left\|y_{1}-y_{2}\right\|\right)<y_{i_{0}}^{*}\left(y_{1}\right),
$$


which gives

$$
s_{y_{i_{0}}^{*}, x}(\gamma) \leq y_{i_{0}}^{*}\left(y_{2}\right)<y_{i_{0}}^{*}\left(y_{1}\right) \leq S_{y_{i_{0}}^{*}, x}(\gamma)
$$

Therefore, $f_{y_{i_{0}}^{*}, x}(\gamma)>0$, which contradicts with the fact $f_{y_{i}^{*}, x} \equiv 0$ on $G \backslash \mathcal{N}$ for each $i \in \mathbb{N}$ and $x \in X$.

\section{Example}

Let $X=\mathbb{R}^{2}$ and $Y=\mathbb{R}^{n}, n \in \mathbb{N}$. Consider the unitary group $U(1)=\{z \in \mathbb{C}:|z|=1\}$ acting on $X$ by rotations around the origin. Identifying $\mathbb{R}^{2}$ with complex numbers field $\mathbb{C}$, we express this action in the form:

$$
z \in U(1): w \in \mathbb{C} \mapsto z w \in \mathbb{C} .
$$

Applying Theorem 2 for Equation (6), we aim to find functions $A$ and $h$ with the properties (7) and (10). Consider an arbitrary function $K:[1, D) \rightarrow c c(Y)$. For any $w \in \mathbb{C} \backslash\{0\}$, we can find unique $n=n(w) \in \mathbb{Z}$ such that

$$
1 \leq \frac{|w|}{D^{n}}<D
$$

Then, define $A_{K}: \mathbb{C} \rightarrow c c(Y)$ as follows:

$$
\begin{array}{r}
A_{K}(w)=D^{2 n(w)} K\left(\frac{|w|}{D^{n(w)}}\right), w \in \mathbb{C} \backslash\{0\} ; \\
A_{K}(w)=\{\overrightarrow{0}\}, w=0 .
\end{array}
$$

Claim 1. Function $A_{K}$ satisfies condition (7). Moreover, for any function $A: \mathbb{C} \rightarrow c c(Y)$ satisfying (7), there exists function $K:[1, D) \rightarrow c c(Y)$ such that $A=A_{K}$.

Proof. Obviously,

$$
\begin{array}{r}
n(D w)=n(w)+1, \\
n(\gamma w)=n(w),
\end{array}
$$

for all $w \in \mathbb{C} \backslash\{0\}, \gamma \in U(1)$. We get

$$
\begin{array}{r}
A_{K}(\gamma w)=D^{2 n(\gamma w)} K\left(\frac{|\gamma w|}{D^{n(\gamma w)}}\right)=D^{2 n(w)} K\left(\frac{|w|}{D^{n(w)}}\right)=A_{K}(w), \\
A_{K}(D w)=D^{2 n(D w)} K\left(\frac{|D w|}{D^{n(D w)}}\right)=D^{2(n(w)+1)} K\left(\frac{|D w|}{D^{n(w)+1}}\right)=D^{2} A_{K}(w) .
\end{array}
$$

for every $w \in \mathbb{C} \backslash\{0\}, \gamma \in U(1)$. These equations evidently hold for $w=0$. Consider an arbitrary function $A$ satisfying $(7)$, and define $K:[1, D) \rightarrow c c(Y)$ as a restriction of $K=\left.A\right|_{[1, D)}$. Then, for any $w \in \mathbb{C} \backslash\{0\}$, we can find unique $\phi \in U(1)$ such that

$$
w=\phi|w|
$$

With regard to the condition (7), we can write

$$
\begin{array}{r}
A(w)=A\left(D^{n(w)} \frac{\phi|w|}{D^{n(w)}}\right)=D^{2 n(w)} A\left(\frac{|w|}{D^{n(w)}}\right)= \\
D^{2 n(w)} K\left(\frac{|w|}{D^{n(w)}}\right)=A_{K}(w),
\end{array}
$$


for all $w \in \mathbb{C} \backslash\{0\}, \gamma \in U(1)$-since

$$
A(0)=\frac{1}{D^{2}} A(D \cdot 0)=\frac{1}{D^{2}} A(0)
$$

and $D>1, A(0)=\{\overrightarrow{0}\}$ must hold, and also $A(0)=A_{K}(0)$.

To construct $b(\gamma, w)$, consider annulus

$$
R=\{w: 1 \leq|w|<D\} \subset X
$$

For any $r \in[1, D)$, we denote

$$
R_{r}=\{w:|w|=r\} \subset R .
$$

Let $f: R \rightarrow Y$ be a function that has the integrable restriction $\left.f\right|_{R_{r}}: R_{r} \rightarrow Y$ for any $r \in[1, D)$. Then, define $b_{f}: U(1) \times \mathbb{C} \rightarrow Y$ as follows:

$$
\begin{array}{r}
b_{f}(\gamma, w)=D^{n(w)} f\left(\frac{w}{D^{n(w)}}\right)-D^{n(w)} f\left(\frac{\gamma w}{D^{n(w)}}\right), \gamma \in U(1), w \in \mathbb{C} \backslash\{0\}, \\
b_{f}(\gamma, w)=\overrightarrow{0}, \gamma \in U(1), w=0 .
\end{array}
$$

Claim 2. Function $b_{f}$ satisfies condition (8). Moreover, for any $b$ satisfying (8), there exists function $f: R \rightarrow Y$ with integrable restrictions to $R_{r}, r \in[1, D)$ such that $b=b_{f}$.

Proof. We can write

$$
\begin{array}{r}
b_{f}(\gamma \sigma, w)-b_{f}(\sigma, w)=D^{n(w)} f\left(\frac{w}{D^{n(w)}}\right)-D^{n(w)} f\left(\frac{\gamma \sigma w}{D^{n(w)}}\right)- \\
D^{n(w)} f\left(\frac{w}{D^{n(w)}}\right)+D^{n(w)} f\left(\frac{\sigma w}{D^{n(w)}}\right)= \\
=D^{n(\sigma w)} f\left(\frac{\sigma w}{D^{n(\sigma w)}}\right)-D^{n(\sigma w)} f\left(\frac{\gamma \sigma w}{D^{n(\sigma w)}}\right)=b_{f}(\gamma, \sigma w), \\
b_{f}(\gamma, D w)=D^{n(D w)} f\left(\frac{D w}{D^{n(D w)}}\right)-D^{n(D w)} f\left(\frac{\gamma D w}{D^{n(D w)}}\right)= \\
=D^{n(w)+1} f\left(\frac{D w}{D^{n(w)+1}}\right)-D^{n(w)+1} f\left(\frac{\gamma D w}{D^{n(w)+1}}\right)=D b_{f}(\gamma, w),
\end{array}
$$

for every $w \in \mathbb{C} \backslash\{0\}, \gamma \in U(1)$. These equations evidently hold for $w=0$.

Consider now an arbitrary function $b$ satisfying (8). Then, each $w \in R$ can be expressed as $w=\phi|w|$, where $\phi \in U(1)$. Define $f: R \rightarrow Y$ such that

$$
f(w)=-b(\phi,|w|), w \in R .
$$

With regard to the condition (8), we can write

$$
\begin{gathered}
b_{f}(\gamma, w)=D^{n(w)} f\left(\frac{w}{D^{n(w)}}\right)-D^{n(w)} f\left(\frac{\gamma w}{D^{n(w)}}\right)= \\
=-D^{n(w)} b\left(\phi, \frac{|w|}{D^{n(w)}}\right)+D^{n(w)} b\left(\gamma \phi, \frac{|\gamma w|}{D^{n(w)}}\right)= \\
=b(\gamma \phi,|w|)-b(\phi,|w|)=b(\gamma, \phi|w|)=b(\gamma, w),
\end{gathered}
$$


for each $w \in \mathbb{C} \backslash\{0\}, \gamma \in U(1)$. Since

$$
b(\gamma, 0)=\frac{1}{D} b(\gamma, D \cdot 0)=\frac{1}{D} b(\gamma, 0)
$$

and $D>1$, we obtain $b(\gamma, 0)=\overrightarrow{0}$, and also $b(\gamma, 0)=b_{f}(\gamma, 0)$.

\section{Conclusions}

In this paper, we have attempted to generalize the study of the following functional equation:

$$
F(x)+\frac{\beta-1}{2 \beta^{2}} F(-\beta x)=\frac{\beta+1}{2 \beta^{2}} F(\beta x),
$$

with $\beta$ natural number, $\beta \neq 1, x \in X,(X,+)$ group uniquely divisible by $\beta$, and $F$ a set-valued mapping from $X$ to the family of non-empty compact, convex subsets of a locally convex linear metric space with an invariant metric. In the article, we focus on the solution of the following integral-functional equation with $D>1$ :

$$
F(x)+\frac{D-1}{D^{2}} \frac{1}{|G|} \int_{G} F(D \sigma x) d \mu(\sigma)=\frac{1}{D} F(D x),
$$

where $G$ is a compact topological group equipped with the right-invariant Haar measure $\mu$.

Author Contributions: Y.B., D.B., and T.Z. conceived the presented ideas on the conceptualization, methodology, and investigation. Writing-review and editing were done by V.B. All authors have read and agreed to the published version of the manuscript.

Funding: The second author was supported by the Project “Long-term development plan of UHK 2020", Czech Republic.

Conflicts of Interest: The authors declare no conflict of interest.

\section{References}

1. Jordan, P.; von Neumann, J. On inner produsts in linear, metric spaces. Ann. Math. 1935, 36, 719-723. [CrossRef]

2. Fréchet, M. Sur la définition axiomatique d'une classe d'eapaces vectoriels distanciés applicables vectoriellement sur l'espace de Hilbert. Ann. Math. 1935, 36, 705-718. [CrossRef]

3. Drygas, H. Quasi-inner products and their applications. In Advances in Multivariate Statistical Analysis, D; Gupta, A.K., Ed.; Reidel Publishing Co.: Dordrecht, The Netherlands, 1987; pp. 13-30.

4. Kannappan, P. Functional Equations and Inequalities with Applications; Springer: New York, NY, USA, 2009.

5. Aczel, J.; Dhombres, J. Functional Equations in Several Variables; Cambridge University Press: New York, NY, USA, 1989.

6. Sikorska, J. On a Method of Solving Some Functional Equations for Set-Valued Functions. Set Valued Var. Anal. 2019, 27, 295-304. [CrossRef]

7. Rådström, H. An embedding theorem for spaces of convex set. Proc. Amer. Math. Soc. 1952, 3, 165-169. [CrossRef]

8. Debreu, G.; Schmeidler, D. The Radon-Nikodym derivative of a correspondence. In Proceedings of the Sixth Berkeley Symposium on Mathematical Statistics and Probability; University of California Press: Berkeley, CA, USA, 1972; Volume 2, pp. 41-56.

Publisher's Note: MDPI stays neutral with regard to jurisdictional claims in published maps and institutional affiliations.

(C) 2020 by the authors. Licensee MDPI, Basel, Switzerland. This article is an open access article distributed under the terms and conditions of the Creative Commons Attribution (CC BY) license (http:/ / creativecommons.org/licenses/by/4.0/). 